\title{
Optimal Orthogonal Basis and Image Assimilation: Motion Modeling
}

\author{
Etienne Huot \\ INRIA $^{1}$, CEREA $^{2}, \mathrm{UVSQ}^{3}$, \\ FRANCE \\ Etienne.Huoteinria.fr
}

\author{
Giuseppe Papari \\ Lithicon Norway AS \\ NORWAY \\ paparielithicon.com
}

\author{
Isabelle Herlin \\ INRIA $^{1}$, CEREA $^{2}$ \\ FRANCE \\ Isabelle.Herlin@inria.fr
}

\begin{abstract}
This paper describes modeling and numerical computation of orthogonal bases, which are used to describe images and motion fields. Motion estimation from image data is then studied on subspaces spanned by these bases. A reduced model is obtained as the Galerkin projection on these subspaces of a physical model, based on Euler and optical flow equations. A data assimilation method is studied, which assimilates coefficients of image data in the reduced model in order to estimate motion coefficients. The approach is first quantified on synthetic data: it demonstrates the interest of model reduction as a compromise between results quality and computational cost. Results obtained on real data are then displayed so as to illustrate the method.
\end{abstract}

\section{Introduction}

Motion estimation from an image sequence is an intensively studied research subject in image processing and computer vision. A powerful class of methods for this task is based on data assimilation (DA), which emerged in this field less than ten years ago [1, 16, 20], after being widely used in remote sensing, geophysical and meteorological applications [4, 14,21]. DA relies on the use of numerical models obtained by discretizing and approximating highly complex and non linear geophysical models. The issue of model reduction, obtained when projecting the dynamic equations on a subspace, arises in a natural way when studying the numerical analysis literature [10, 17]. For instance, D'Adamo et al. [5] apply model reduction for estimating flow dynamics from particle image velocimery measures with a data assimilation method. Herlin et al. [9] and Drifi et al. [8] describe motion estimation on long temporal sequences with reduced models obtained by Proper Order

\footnotetext{
${ }^{1}$ Institut National de Recherche en Informatique et Automatique

${ }^{2}$ CEREA, joint laboratory École des Ponts ParisTech - EDF R\&D, Université Paris-Est

${ }^{3}$ Université de Versailles - Saint-Quentin-en-Yvelines
}

Decomposition. However, their reduction method does not allow to either constrain properties on motion fields or to apply specific boundary conditions.

In this paper, a novel projection basis is proposed (Section 3), which is derived by an optimality criterion, that takes into account the shape of the basin in which the water flows, and some desirable properties of the concerned motion, such as smoothness and zero divergence. These waveforms are applied, by means of a Galerkin projection, to obtain a reduced model of the fluid dynamic system under study which is used for image assimilation (Section 21. Experimental results and conclusions are respectively presented in Sections 4 and 5

\section{Model reduction and data assimilation}

In this section, the full and reduced model of the physical system under study are reviewed (Subsection 2.1), and the variational approach to image assimilation is described (Subsection 2.2).

\subsection{Dynamic model}

In the following, a numerical procedure for the solution of the fluid dynamic equations is presented, which is based on the Galerkin projection of the state vector on motion and image subspaces spanned by given families of projection functions.

Let $A=\Omega \times[0, T]$ be a bounded space-time domain on which images and motion fields are defined, and let $(\mathbf{r}, t)$ be a point of $\Omega$.

In this paper, processed images are Sea Surface Temperature data acquired over oceans. As the concern is motion estimation from these data, the state vector $\mathbf{X}$ is composed of a $2 \mathrm{D}$ vector field $\mathbf{w}(\mathbf{r})$, which represents the horizontal velocity of the water, and a scalar field $I_{s}(\mathbf{r})$, which represents the surface temperature of the water. The latter is also called pseudo-image in the image assimilation literature. In other words, we have $\mathbf{X}=\left(\begin{array}{ll}\mathbf{w}^{T} & I_{s}\end{array}\right)^{T}$. The purpose of the pseudo-image is to allow an easy comparison between the state vector and the real image observations: the pseudoimage values have to be close to the real ones. The model 
used to describe the temporal evolution assumes that the velocity field is self-advected and pseudo-images are advected by motion:

$$
\left\{\begin{array}{l}
\frac{\partial \mathbf{w}}{\partial t}(\mathbf{r}, t)+(\mathbf{w} \cdot \nabla) \mathbf{w}(\mathbf{r}, t)=0 \\
\frac{\partial I_{s}}{\partial t}(\mathbf{r}, t)+\mathbf{w} \cdot \nabla I_{s}(\mathbf{r}, t)=0
\end{array}\right.
$$

System (1) can be rewritten as the evolution equation of a state vector $\mathbf{X}$ :

$$
\frac{\partial \mathbf{X}}{\partial t}(\mathbf{r}, t)+\mathbb{M}(\mathbf{X})(\mathbf{r}, t)=0
$$

where the evolution model $\mathbb{M}$ includes the advection terms $(\mathbf{w} \cdot \nabla) \mathbf{w}$ and $\mathbf{w} \cdot \nabla I_{s}$. This equation is now projected into the subspaces spanned by the orthogonal bases $\Phi=$ $\left\{\phi_{i}(\mathbf{r})\right\}_{i=1 \ldots K}$ and $\Psi=\left\{\psi_{j}(\mathbf{r})\right\}_{j=1 \ldots L}$, which are defined to represent motion and images on the domain $\Omega$. In (1), w and $I_{s}$ are replaced by their projections on these subspaces:

$$
\begin{aligned}
\mathbf{w}(\mathbf{r}, t) & \approx \sum_{i=1}^{K} a_{i}(t) \phi_{i}(\mathbf{r}) \\
I_{s}(\mathbf{r}, t) & \approx \sum_{j=1}^{L} b_{j}(t) \psi_{j}(\mathbf{r})
\end{aligned}
$$

Using linearity properties, we get :

$$
\left\{\begin{array}{l}
\sum_{i=1}^{K} \frac{d a_{i}}{d t} \phi_{i}(\mathbf{r})+\sum_{i, j=1}^{K} a_{i} a_{j}\left(\phi_{i}(\mathbf{r}) \cdot \nabla\right) \phi_{j}(\mathbf{r})=0 \\
\sum_{j=1}^{L} \frac{d b_{j}}{d t} \psi_{j}(\mathbf{r})+\sum_{i, j=1}^{K, L} a_{i} b_{j} \phi_{i}(\mathbf{r}) \cdot \nabla \psi_{j}(\mathbf{r})=0
\end{array}\right.
$$

The first equation is projected on $\phi_{k}$, for $k=1 \ldots K$, and the second one on $\psi_{l}$, for $l=1 \ldots L$. As $\Phi$ are $\Psi$ are orthogonal bases, we obtain:

$$
\left\{\begin{array}{l}
\frac{d a_{k}}{d t}\left\langle\phi_{k}, \phi_{k}\right\rangle+\sum_{i, j=1}^{K} a_{i} a_{j}\left\langle\left(\phi_{i} \cdot \nabla\right) \phi_{j}, \phi_{k}\right\rangle=0, \\
\frac{d b_{l}}{d t}\left\langle\psi_{l}, \psi_{l}\right\rangle+\sum_{i, j=1}^{K, L} a_{i} b_{j}\left\langle\phi_{i} \cdot \nabla \psi_{j}, \psi_{l}\right\rangle=0
\end{array}\right.
$$

with $\langle\cdot, \cdot\rangle$ denoting the scalar product of the motion and image subspaces, defined by: $\langle f, g\rangle=\int f(\mathbf{r}) g(\mathbf{r}) d \mathbf{r}$. By respectively dividing equations by $\left\langle\phi_{k}, \phi_{k}\right\rangle$ and $\left\langle\psi_{l}, \psi_{l}\right\rangle$, it comes:

$$
\left\{\begin{array}{l}
\frac{d a_{k}}{d t}+\sum_{i, j=1}^{K} a_{i} a_{j} \frac{\left\langle\left(\phi_{i} \cdot \nabla\right) \phi_{j}, \phi_{k}\right\rangle}{\left\langle\phi_{k}, \phi_{k}\right\rangle}=0 \\
\frac{d b_{l}}{d t}+\sum_{i, j=1}^{K, L} a_{i} b_{j} \frac{\left\langle\phi_{i} \cdot \nabla \psi_{j}, \psi_{l}\right\rangle}{\left\langle\psi_{l}, \psi_{l}\right\rangle}=0
\end{array}\right.
$$

Let define:

- $a(t)=\left(\begin{array}{lll}a_{1}(t) & \ldots & a_{K}(t)\end{array}\right)^{T}$ the vector of coefficients, obtained when projecting $\mathbf{w}(t)$ on the basis $\Phi$,

- $b(t)=\left(\begin{array}{lll}b_{1}(t) & \ldots & b_{L}(t)\end{array}\right)^{T}$, the vector of coefficients, obtained when projecting $I_{s}(t)$ on the basis $\Psi$,

- $B(k)$ the $K \times K$ matrix whose $(i, j)$ element is:

$$
B(k)_{i, j}=\frac{\left\langle\left(\phi_{i} \cdot \nabla\right) \phi_{j}, \phi_{k}\right\rangle}{\left\langle\phi_{k}, \phi_{k}\right\rangle},
$$

- $G(l)$ the $K \times L$ matrix whose $(i, j)$ element is:

$$
G(l)_{i, j}=\frac{\left\langle\phi_{i} \cdot \nabla \psi_{j}, \psi_{l}\right\rangle}{\left\langle\psi_{l}, \psi_{l}\right\rangle}
$$

System (6) rewrites:

$$
\left\{\begin{array}{l}
\frac{d a_{k}}{d t}(t)+a(t)^{T} B(k) a(t)=0, \quad k=1 \ldots K \\
\frac{d b_{l}}{d t}(t)+a(t)^{T} G(l) b(t)=0, \quad l=1 \ldots L
\end{array}\right.
$$

A reduced state vector is defined as $\mathbf{X}_{R}(t)=\left(\begin{array}{l}a(t) \\ b(t)\end{array}\right)$, and System (7) is summarized by:

$$
\frac{d \mathbf{X}_{R}}{d t}(t)+\mathbb{M}_{R}\left(\mathbf{X}_{R}(t)\right)=0
$$

the reduced model $\mathbb{M}_{R}$ being the Galerkin projection of the full model $\mathbb{M}$ on bases $\Phi$ and $\Psi$.

\subsection{Data assimilation}

Motion estimation will be obtained by means of data assimilation in the reduced model described in the previous subsection, where the reduced state vector $\mathbf{X}_{R}$ satisfies the evolution equation (8). Observations, denoted by the vector $\mathbf{Y}(t)$, are linked to the state vector by an observation operator $\mathbb{H}$ :

$$
\mathbf{Y}(t)=\mathbb{H} \mathbf{X}_{R}(t)+\epsilon_{R}(t)
$$

where $\mathbb{H}$ is the projection operator that maps $\mathbf{X}_{R}(t) \rightarrow b(t)$. The observation error $\epsilon_{R}(t)$ represents uncertainty on the observations and on the state vector values. Some heuristics are usually available on the value of the state vector at date 0 . This is described by the background value $\mathbf{X}_{R b}$ of the state vector:

$$
\mathbf{X}_{R}(0)=\mathbf{X}_{R b}+\epsilon_{B}
$$

The background error $\epsilon_{B}$ describes the uncertainty on the background value. The variables $\epsilon_{R}(t)$ and $\epsilon_{B}$ are Gaussianly distributed with zero-mean and covariance matrices $R$ and $B$ respectively. For a posteriori estimation of 
$\mathbf{X}_{R}(0)$ given the observations, the following cost functional $J$ needs to be minimized:

$$
\begin{array}{r}
J\left(\mathbf{X}_{R}(0)\right)=\int_{A}\left(\mathbf{Y}-\mathbb{H} \mathbf{X}_{R}\right)^{T} R^{-1}\left(Y-\mathbb{H} \mathbf{X}_{R}\right) \\
\quad+\int_{\Omega}\left(\mathbf{X}_{R}(0)-\mathbf{X}_{R b}\right)^{T} B^{-1}\left(\mathbf{X}_{R}(0)-\mathbf{X}_{R b}\right)
\end{array}
$$

In order to apply the dual method described in [14], an adjoint variable $\lambda$ is introduced, which is defined by the following equations:

$$
\begin{aligned}
\lambda(T) & =0 \\
-\frac{d \lambda}{d t}+\left(\frac{\partial \mathbb{M}_{R}}{\partial \mathbf{X}_{R}}\right)^{*} \lambda & =\mathbb{H}^{T} R^{-1}\left(\mathbf{Y}-\mathbb{H} \mathbf{X}_{R}\right)
\end{aligned}
$$

where $\left(\frac{\partial \mathbb{M}_{R}}{\partial \mathbf{X}_{R}}\right)^{*}$ denotes the adjoint model of $\mathbb{M}_{R}$. The gradient of $J$, denoted $\nabla J$, is derived with a variation method [15], which yields:

$$
\nabla J\left(\mathbf{X}_{R}(0)\right)=B^{-1}\left(\mathbf{X}_{R}(0)-\mathbf{X}_{R b}\right)+\lambda(0)
$$

The cost function $J$ is then minimized using a steepest descent method. At each iteration, the forward time integration of $\mathbf{X}_{R}$ is performed, according to Equation (8), and is used to compute the value of $J$ in Equation (11); then a backward integration of $\lambda$, according to Equation (13), provides the value $\lambda(0)$ that is used to compute $\nabla J$ in Equation (14). An efficient solver [2] is applied to perform the optimization given values of $J$ and $\nabla J$.

\section{Definition of optimal bases}

In this section, the proposed projection bases for motion and image spaces are introduced as solutions of an ad hoc constrained minimization problem. The minimization problem is first formulated in general terms (Subsection 3.1), and it is then particularized to the case of motion estimation (Subsection 3.2).

\subsection{Continuous formulation of the projection basis}

Let $\mathfrak{F}$ be a Hilbert functional space, with given inner product $\langle\cdot, \cdot\rangle$. Let $\mathcal{Q}: \mathfrak{F} \rightarrow \mathbb{R}$ be a positively quadratic functional expressed as $\mathcal{Q}(f)=\langle\mathcal{L}(f), f\rangle$, with $\mathcal{L}$ a selfadjoint linear operator. The operator $\mathcal{L}$ coincides with the gradient of $\mathcal{Q}$.

Let denote $\boldsymbol{\psi}_{1}, \ldots, \boldsymbol{\psi}_{n}$ the optimal waveforms, that are solutions of the following constrained minimization problem:

$$
\left\{\begin{array}{l}
\min _{\left(\boldsymbol{\psi}_{1}, \ldots, \boldsymbol{\psi}_{n}\right) \in \mathfrak{F}^{n}} \sum_{k=1}^{n} \mathcal{Q}\left(\boldsymbol{\psi}_{k}\right) \\
\mathcal{B}\left(\boldsymbol{\psi}_{k}\right)=0, \quad k=1, \ldots, n \\
\left\langle\boldsymbol{\psi}_{j}, \boldsymbol{\psi}_{k}\right\rangle=\delta_{j, k}
\end{array}\right.
$$

where $\mathcal{B}$ is a linear operator on $\mathfrak{F}$ and $\delta_{j, k}$ is the delta Kronecker symbol.

Let $\mathfrak{F}_{\mathcal{B}} \subset \mathfrak{F}$ denote the null space of $\mathcal{B}$ and $\mathcal{L}_{\mathcal{B}}$ be the restriction of $\mathcal{L}$ on this subspace.

Two theorems are at hand in order to compute the solution $\left(\boldsymbol{\psi}_{1}, \ldots, \boldsymbol{\psi}_{n}\right)$.

Theorem 1. The waveforms $\psi_{1}, \ldots, \psi_{n}$ obtained from (15) are the eigenfunctions of $\mathcal{L}_{\mathcal{B}}$ that correspond to its $n$ smallest eigenvalues.

Theorem 2. The waveforms $\psi_{1}, \ldots, \psi_{n}$ that arise from (15) coincide with the $n$ first solutions of the following progressive unbounded minimization problem:

$$
\begin{cases}\boldsymbol{\psi}_{1}=\arg \min _{\boldsymbol{\psi} \in \mathcal{F}_{\mathcal{B}}} \mathcal{Q}_{\mathcal{B}}(\boldsymbol{\psi}), \quad|\boldsymbol{\psi}|^{2}=1 \\ \boldsymbol{\psi}_{2}=\arg \min _{\boldsymbol{\psi} \in \mathfrak{F}_{\mathcal{B}}} \mathcal{Q}_{\mathcal{B}}(\boldsymbol{\psi}), \quad|\boldsymbol{\psi}|^{2}=1, \quad \boldsymbol{\psi} \perp \boldsymbol{\psi}_{1} \\ \ldots \\ \boldsymbol{\psi}_{k}=\arg \min _{\boldsymbol{\psi} \in \mathfrak{F}_{\mathcal{B}}} \mathcal{Q}_{\mathcal{B}}(\boldsymbol{\psi}), \quad|\boldsymbol{\psi}|^{2}=1, \quad \boldsymbol{\psi} \perp \boldsymbol{\psi}_{j}, \quad \forall j<k \\ \ldots\end{cases}
$$

In practice, the proposed waveforms are computed as eigenfunctions of a symmetric square matrix which is calculated from the discretized versions of the operators $\mathcal{L}$ and $\mathcal{B}$. Moreover, in the context of motion estimation $\mathcal{L}$ and $\mathcal{B}$ are differential operators. Therefore, the resulting matrix will be sparse, thus reducing computational complexity.

\subsection{Definition of bases for motion estimation}

This subsection describes the definition of scalar and vector bases, obtained with the method described above, for two space domains $\Omega$ : the whole Black Sea basin is used to demonstrate the ability of the method to deal with complex regions, and a rectangular domain is considered, on which the data assimilation method is tested in order to quantify the approach with synthetic data. Given the definition of $\Omega$, the bases are depending on the choice of the functional $\mathcal{Q}$ and operator $\mathcal{B}$, which have to be defined in order to ensure the required properties of image and motion fields.

As to the scalar waveforms that we use for image subspace, we consider solutions of the minimization problem 16, with:

$$
\mathcal{Q}(\psi) \triangleq \int_{\Omega}|\nabla \psi(\mathbf{r})|^{2} \mathrm{~d} \mathbf{r}
$$

for $\boldsymbol{\psi}: \Omega \rightarrow \mathbb{R}$ being a scalar vector field and $\mathcal{B}(\psi)$ applying Neumann boundary conditions on image data. Examples of the resulting waveforms are displayed in Fig. 1 . In Fig. 2. reconstructions obtained with bases of 50, 100 and 500 elements are displayed. As we see, the more the number of terms increases the more finer scales are present in the approximation. Moreover, it is possible to define the 

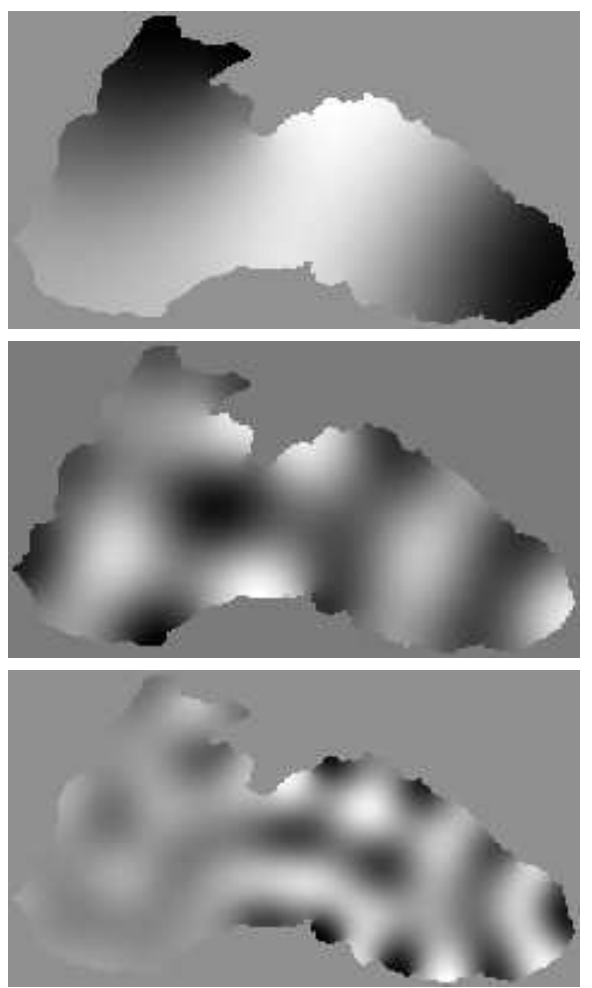

Figure 1. Eigenfunctions $\psi_{n}$, with Neumann Boundary conditions, for $n=4, n=20$ and $n=50$.

number of elements according to the size of studied structures on images.

As to vector waveforms related to the motion subspace, we will still consider solutions of the minimization problem (16), with:

$$
\mathcal{Q}(\phi) \triangleq \int_{\Omega}|\nabla \phi(\mathbf{r})|^{2} \mathrm{~d} \mathbf{r}
$$

where $\phi: \Omega \rightarrow \mathbb{R}^{2}$ is a planar vector field. As to the operator $\mathcal{B}(\phi)$, different situations may be defined. In this paper, we consider divergence-free motion with Dirichlet boundary conditions, thus having:

$$
\mathcal{B}(\phi)(\mathbf{r})= \begin{cases}\mathbf{n}(\mathbf{r}) \cdot \boldsymbol{\phi}(\mathbf{r}), & \mathbf{r} \in \partial \Omega \\ \operatorname{div} \phi(\mathbf{r}), & \text { otherwise }\end{cases}
$$

Examples of the resulting functions are shown in Fig. 3, in which vector fields are represented by streamlines.

The projection bases proposed in this subsection have some similarity with the wavelets described in [12, 13], which have been used for optical flow estimation [6, 7]. Specifically, a family of bi-orthogonal diadic projection functions is derived in [12,13] as the curl of standard biorthogonal wavelets, on a square domain. Several mathematical properties of those wavelets are described: the curl of these bi-orthogonal wavelets are still bi-orthogonal, and they satisfy the Dirichlet conditions on the boundaries of the
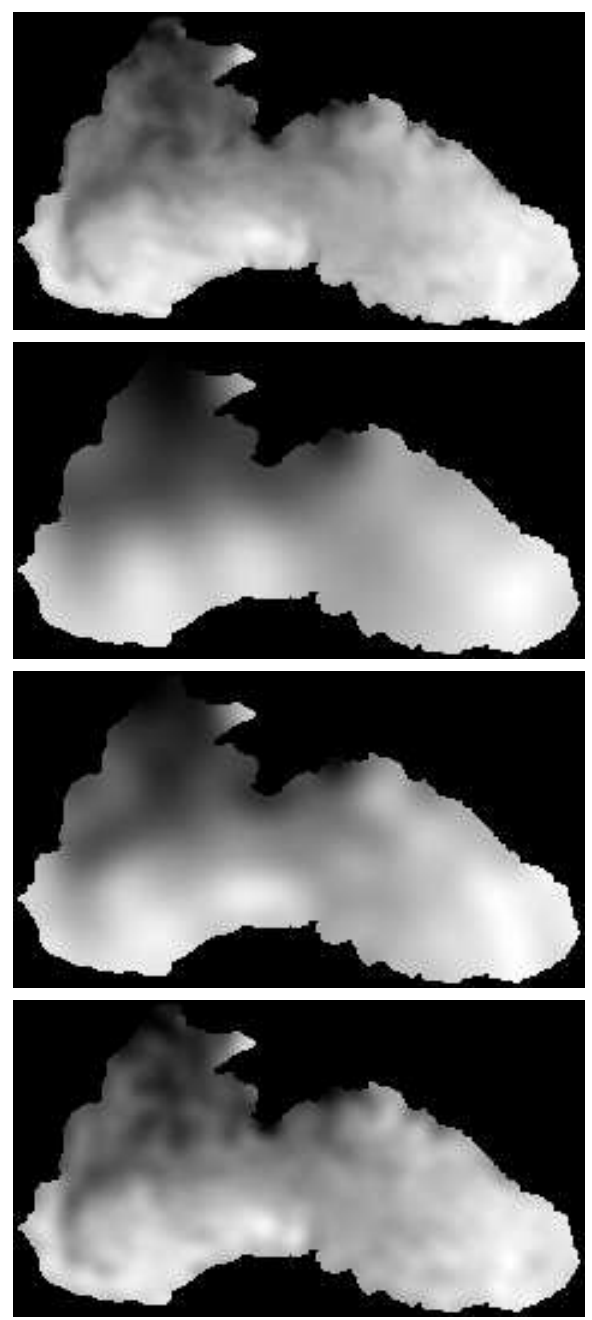

Figure 2. Top to bottom: A satellite image and its reconstruction with 50, 100, and 500 elements respectively.

square. However, unlike the projection basis proposed here, the wavelets proposed in [12,13] are not suitable to represent vector fields that are defined on an irregular domain $\Omega$, since the boundary conditions are not met on the boundary $\partial \Omega$ in the case where $\Omega$ is not square. Specifically, if a given velocity field $\mathbf{w}(\mathbf{r})$ is projected onto a complex domain, the error $\epsilon^{2}=\int_{\partial \Omega}(\mathbf{n}(\mathbf{r}) \cdot \mathbf{w}(\mathbf{r}))^{2} \mathrm{~d} \mathbf{r}$ will be in general nonzero, where $\mathbf{n}(\mathbf{r})$ is the orthogonal unit vector to $\partial \Omega$. In order to keep $\epsilon$ small, a large number of coefficients is needed in the expansion of $\mathbf{w}(\mathbf{r})$, which would make the model sensitive to noise and memory requirement and computation time not suitable for users. Moreover, even if the value of $\epsilon$ is sufficiently small in the initial condition, it is not guaranteed that it will stay small when a simulation is run with the reduced model. As well known, errors in the boundary condition cause the simulation to become unstable after a certain number of time steps. To our knowledge, this is the most serious limitation of the wavelet proposed 

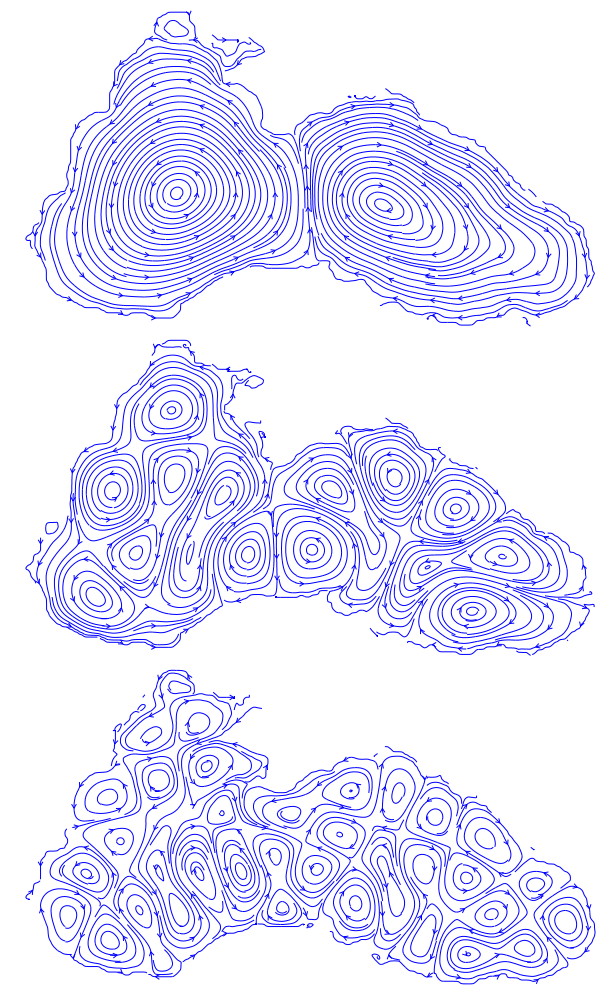

Figure 3. Elements 1, 20 and 50 of the divergence-free motion basis.

by [12,13], which is overcome by the projection basis proposed in this article. In addition, in [12, 13] only vector waveforms are proposed, therefore their framework cannot be used to represent the pseudo-image component of the state vector. In contrast, the framework presented here can be used both for vector and scalar components of the state vector.

\section{Results on motion estimation}

The reduced model obtained with the scalar basis for images and the divergence-free basis for motion fields has been used for estimating motion on a sequence of six satellite images acquired by NOAA/AVHRR sensors on May 14th and 15th 2005. Three acquisitions are displayed on Fig. 4. The number of elements for the scalar basis is 240 and the one of the vector basis is 24 . Four elements are displayed on Fig. 5 for images and on Fig. 6 for motion fields.

As no ground-truth is available for satellite data, a twinexperiment is first defined in order to quantify the method. A simulation is performed with the motion field displayed in Fig. 7, top, and the first satellite image of Fig. 4 as initial conditions. For a clearer rendering of the motion, arrows that represent the velocity field are superposed to the usual color representation. Statistics of that motion field are given in Table 1 1 minimal, maximal and average values

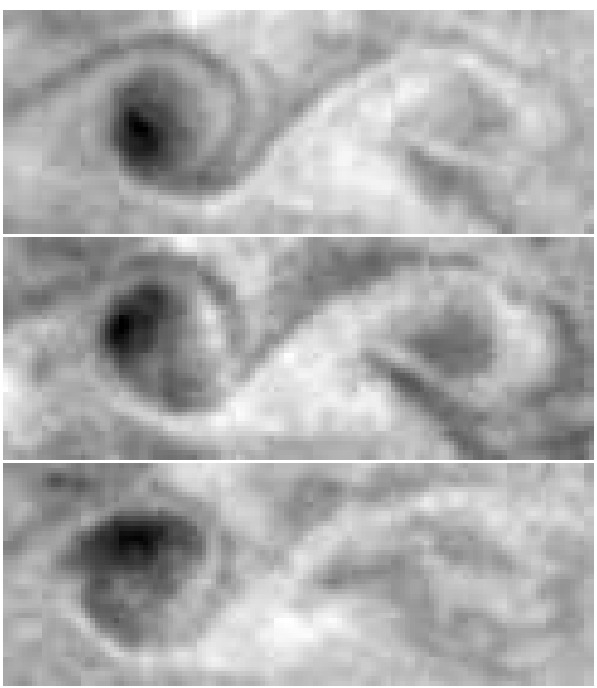

Figure 4. Images 1, 3 and 6 of the satellite sequence.
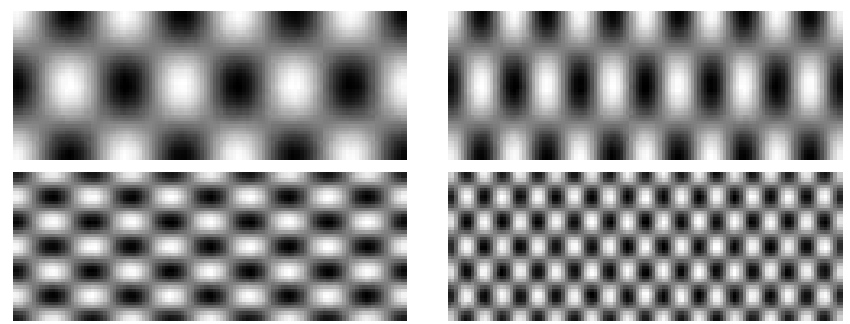

Figure 5. Four elements $\psi_{n}$ of the scalar basis, for $n=30, n=$ $60, n=120$, and $n=240$.
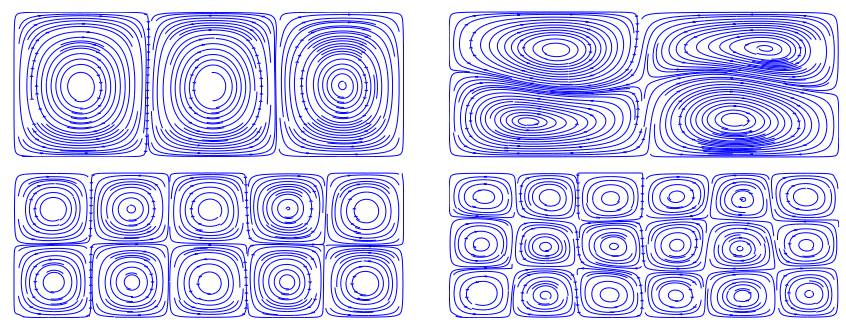

Figure 6. Four elements $\psi_{n}$ of the motion basis, for $n=3, n=6$, $n=12$, and $n=24$.

\begin{tabular}{|c|c|c|c|}
\hline & $\min$ & avg & $\max$ \\
\hline \hline magnitude (m/s) & $3.6 \mathrm{e}-04$ & $1.4 \mathrm{e}-01$ & $3.5 \mathrm{e}-01$ \\
\hline
\end{tabular}

Table 1. Statistics on the norm of the initial motion field.

of the norm. The simulation provides a sequence of six pseudo-images, taken at same dates than the real acquisitions. These pseudo-images are then assimilated with the reduced model in order to estimate the underlying motion. Value at date 0, named estimation, is displayed in Fig. 7. bottom. The method is further compared with four stateof-the-art methods and results are also given in Fig. 7 The error between motion estimations and ground-truth is quantified in Table 2. The state-of-the-art methods rely either on 


\begin{tabular}{|c|ccc|ccc|}
\hline & \multicolumn{3}{|c|}{ Magnitude error $(\mathrm{m} / \mathrm{s})$} & \multicolumn{3}{|c|}{ Angular error in degrees } \\
Method & min & avg & max & min & avg & max \\
\hline \hline Horn et al $[11]$ & $2.0 \mathrm{e}-06$ & $6.9 \mathrm{e}-02$ & $3.5 \mathrm{e}-01$ & $1.1 \mathrm{e}-03$ & 34 & 180 \\
Suter $[19]$ & $0.0 \mathrm{e}+00$ & $6.3 \mathrm{e}-02$ & $2.7 \mathrm{e}-01$ & $3.6 \mathrm{e}-03$ & 37 & 180 \\
Corpetti et al $[3]$ & $6.0 \mathrm{e}-06$ & $7.3 \mathrm{e}-02$ & $2.5 \mathrm{e}-01$ & $5.6 \mathrm{e}-03$ & 32 & 180 \\
Sun et al $[18]$ & $5.4 \mathrm{e}-05$ & $6.3 \mathrm{e}-02$ & $2.8 \mathrm{e}-01$ & $1.2 \mathrm{e}-03$ & 22 & 180 \\
Our approach & $0.0 \mathrm{e}+00$ & $2.8 \mathrm{e}-04$ & $1.7 \mathrm{e}-03$ & $2.3 \mathrm{e}-04$ & 19 & 58 \\
\hline
\end{tabular}

Table 2. Error analysis: misfit between motion results and ground-truth.

a $L_{2}$ regularisation of motion [11, 18] or on a second-order regularisation of the divergence [3, 19]. In order to better visualize the differences between methods, we defined five characteristics points on the first observation, which are displayed as red crosses on Fig. 8 These characteristic points are then advected by the ground-truth motion field (displayed in red), the one obtained with our method (displayed in green), and the result of Sun et al. [18] (displayed in blue), that has been identified as the best one by statistics. Trajectories obtained with the two first follow Equation (8), while the trajectory obtained by Sun is piecewise linear. The characteristic points obtained at the end of the whole advection process are visualized on the last observation on Fig. 9. The color of the ellipse surrounding each set of points gives an additional information on the quality of the result: a green ellipse codes that our method gives the best result, while a blue one means that Sun's algorithm provides a better result.

The sequence of real satellite observations partly displayed on Fig. 4 has then been processed for estimating its motion with the reduced model. Result is given on the bottom part of Fig. 10. An additional experiment has been conducted with a scalar basis of size 120 . This demonstrates that if small scales of image data are not taken into account in the basis, motion can not be correctly retrieved. On the other hand, experiment with a scalar basis of size 480 demonstrates that further increasing the subspace dimension does not allow to improve results and has for consequence to increase the size of the reduced model and computational cost. The right number of elements should be chosen accordingly to the size of the spatial structures that impact motion. The middle part of Fig. 10 displays the result obtained when satellite images are assimilated with the full model described by System (11). As the full model considers a local description of motion, under the divergencefree constraint, global characteristics such as the two main vortices are underestimated, due to smoothing created by the data assimilation process. On the other hand, having determined the size of motion basis in accordance to the minimal size of structures to be retrieved allows a better modeling and a good retrieval of these vortices. The characteristic points of Fig. 8 have been advected by Sun motion field (displayed on top of Fig. 10, the full model (middle of
Fig. 10) and our approach (bottom of Fig. 10). Positions on the last observation are displayed on Fig. 11. blue for Sun, yellow for full model and green for our approach.

Figure 12 displays a second satellite sequence of Sea Surface Temperature images acquired on July 27th and 28th 2007. Figure 13 provides results of motion estimation: Sun et al. on the top, full model in the middle, and our method on the bottom. Again, it can be observed that structures are better assessed and retrieved by the reduced model.

\section{Conclusion and Perspectives}

The main contribution of the paper is the new set of orthogonal projection functions introduced for reduced models. The new waveforms have been obtained by maximizing smoothness while imposing desirable properties, such as zero divergence and suitable boundary conditions. The proposed waveforms enable us to write down a reduced model, which has been used for image assimilation. As we see from the experimental results, structures are better assessed with our approach, while computational cost becomes low, even for large size basins.

A major perspective of this work concerns the improvement of the properties imposed to the image and motion bases. As to scalar waveforms, for the image subspace, constraints to allow finer scales at positions of fine structures will be investigated from a long term satellite images data base. As to vector waveforms, for the motion subspace, we will investigate how to use a data base of analysis, obtained by data assimilation in an oceanographic model, to derive properties to be imposed to the basis elements.

\section{References}

[1] D. Béréziat and I. Herlin. Solving ill-posed image processing problems using data assimilation. Numerical Algorithms, 52(2):219-252, 2011.

[2] R. H. Byrd, P. Lu, and J. Nocedal. A limited memory algorithm for bound constrained optimization. Journal on Scientific and Statistical Computing, 16(5):1190-1208, 1995.

[3] T. Corpetti, E. Mémin, and P. Prez. Dense estimation of fluid flows. IEEE Transactions on Pattern Analysis and Machine Intelligence, 24(3):365-380, Mar. 2002.

[4] P. Courtier, J.-N. Thépaut, and A. Hollingsworth. A strategy for operational implementation of $4 \mathrm{~d}$-var, using and incre- 


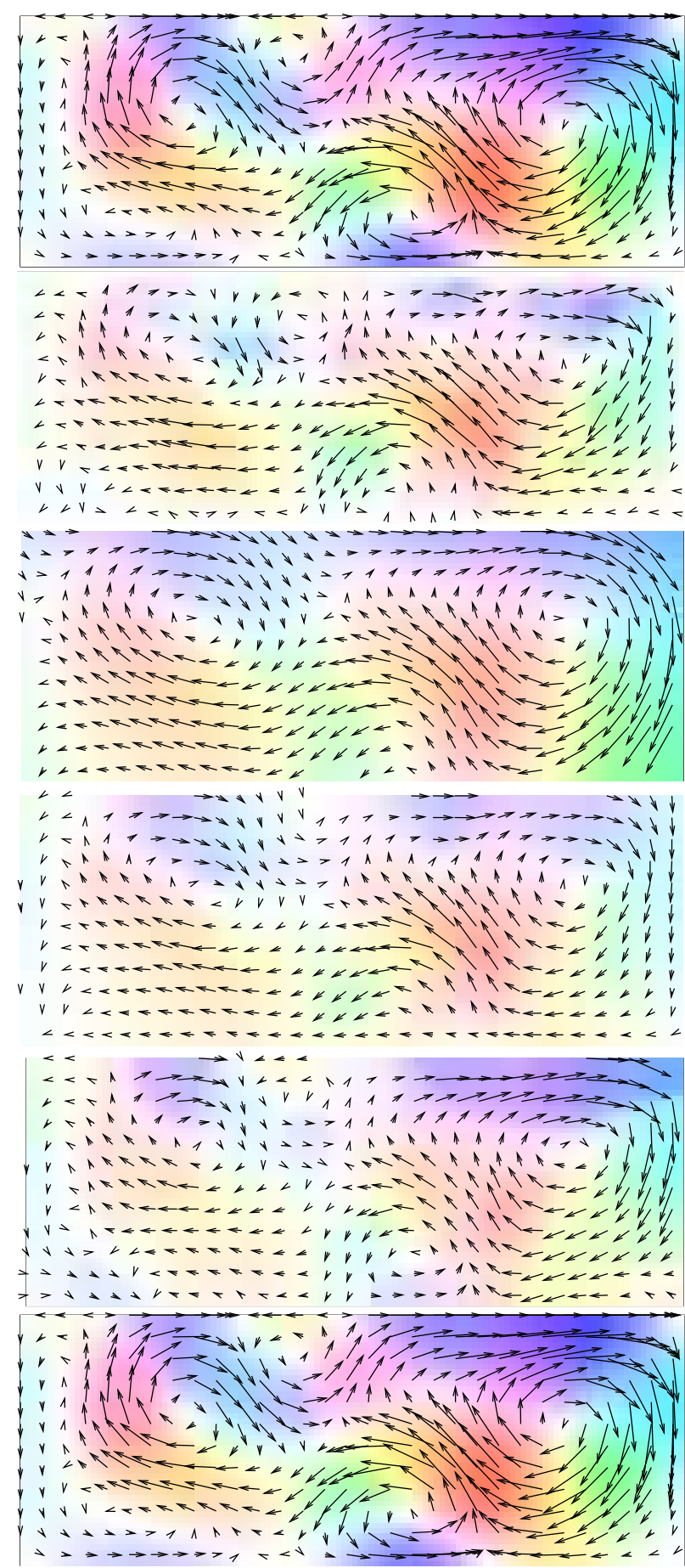

Figure 7. Result of the twin experiment. From top to bottom: ground-truth, Horn et al. [11], Suter [19], Corpetti et al. [3], Sun et al. [18], and our estimation.

mental approach. Quaterly Journal of the Royal Meteorological Society, 120(1367-1387), 1994.

[5] J. D'Adamo, N. Papadakis, E. Mémin, and A. G. Variational assimilation of POD low-order dynamical systems. Journal of Turbulence, 8(9):1-22, 2007.

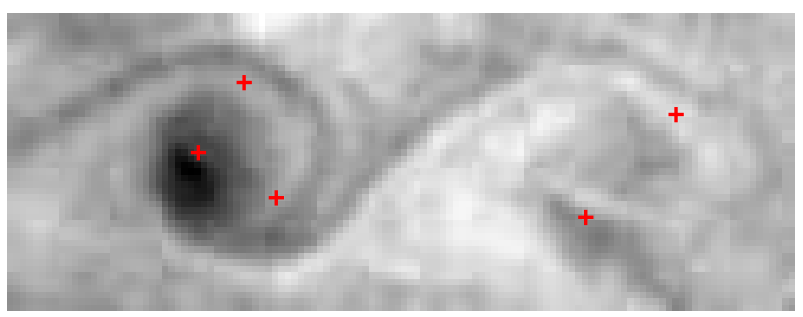

Figure 8. Characteristic points.

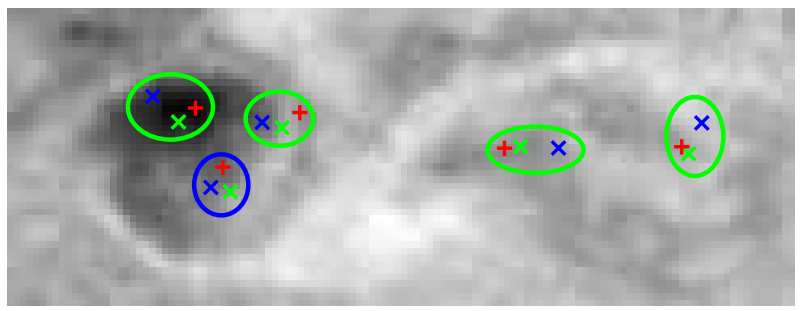

Figure 9. Characteristic points on the last observation.
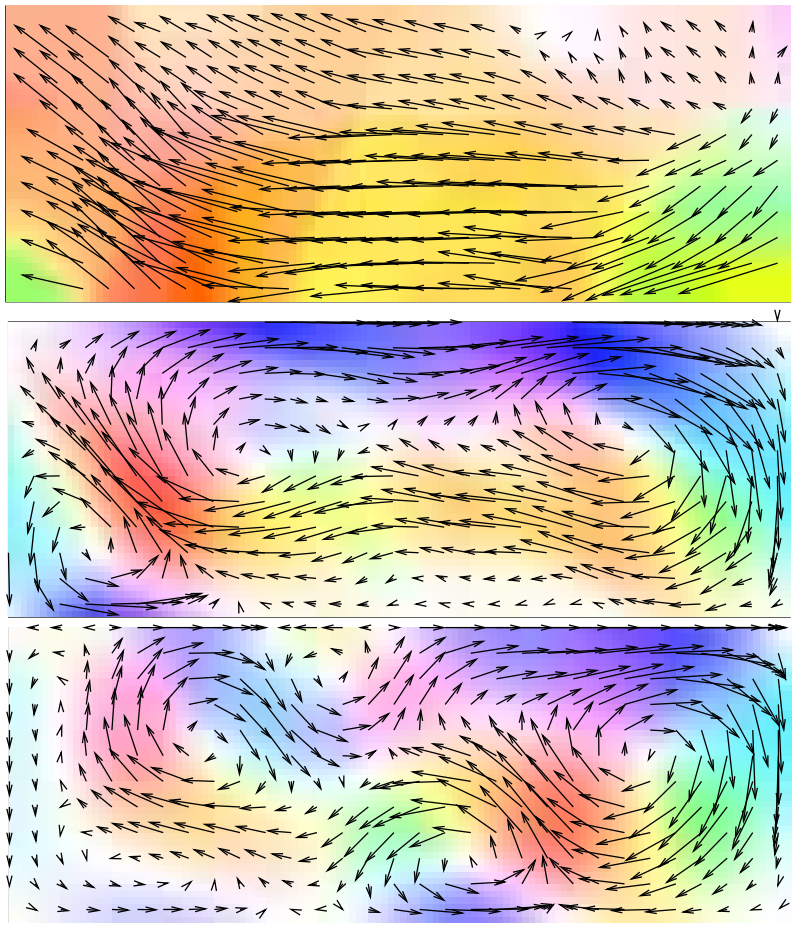

Figure 10. Result on real data. Top: Sun et al method [18]. Middle: using the full model. Bottom: our estimation.

[6] P. Dérian, P. Héas, C. Herzet, and E. Mémin. Wavelets to reconstruct turbulence multifractals from experimental image sequences. In 7th International Symposium on Turbulence and Shear Flow Phenomena, TSFP-7, Ottawa, Canada, July 2011.

[7] P. Dérian, P. Héas, C. Herzet, and E. Mémin. Wavelets and optical flow motion estimation. Numerical Mathematics: Theory, Methods and Applications, 2012.

[8] K. Drifi and I. Herlin. Coupling reduced models for opti- 


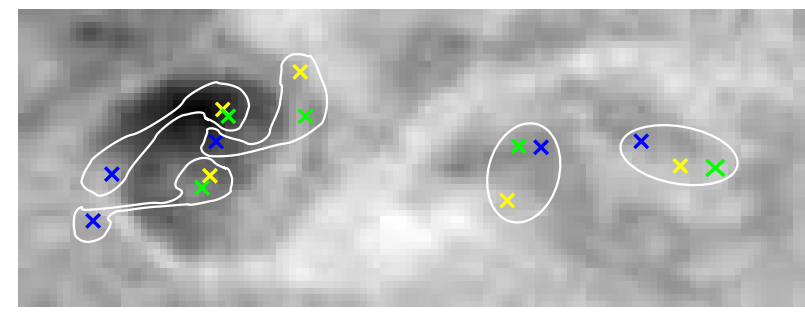

Figure 11. Characteristic points on the last observation.
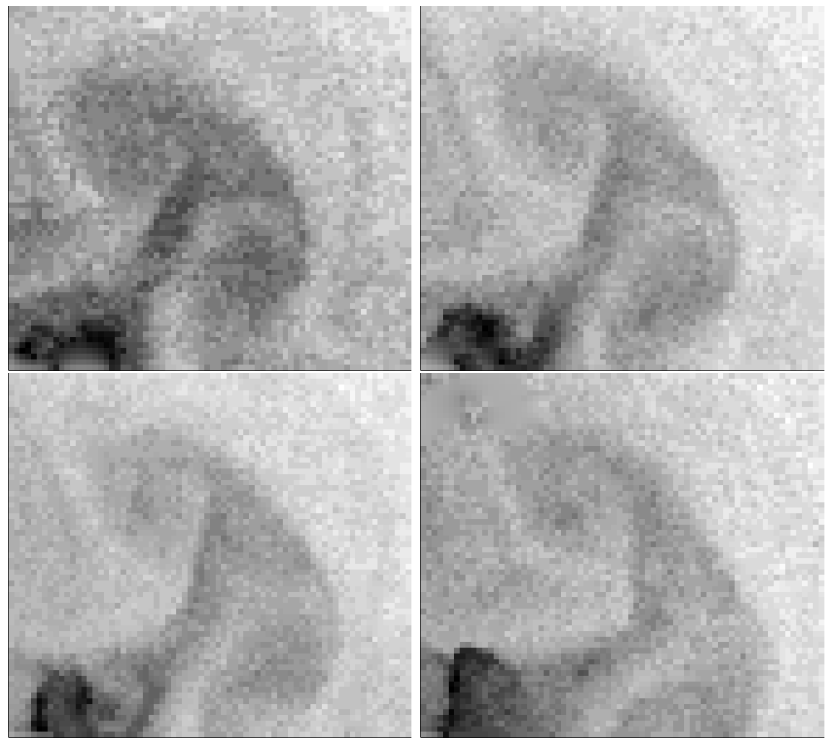

Figure 12. Second satellite sequence: top to bottom and left to right.

mal motion estimation. In Proceedings of International Conference on Pattern Recognition (ICPR), pages 2651-2654, Tsukuba, Japan, November 2012.

[9] I. Herlin and K. Drifi. Learning reduced models for motion estimation on long temporal image sequences. In Proceedings of IEEE International Geoscience and Remote Sensing Symposium (IGARSS), Munich, Germany, July 2012.

[10] C. Homescu, L. R. Petzold, and R. Serban. Error estimation for reduced-order models of dynamical systems. SIAM Journal of Numerical Analysis, 43(4):1,693-1,714, 2005.

[11] B. Horn and B. Schunk. Determining optical flow. Artificial Intelligence, 17:185-203, 1981.

[12] S. Kadri Harouna, P. Dérian, P. Héas, and E. Mémin. Divergence-free wavelets and high order regularization. International Journal of Computer Vision, 2012.

[13] S. Kadri Harouna and V. Perrier. Effective construction of divergence-free wavelets on the square. Journal of Computational and Applied Mathematics, 240:74-86, March 2012.

[14] F. Le Dimet and O. Talagrand. Variational algorithms for analysis and assimilation of meteorological observations: theoretical aspects., pages 97-110. Tellus, 1986.

[15] J.-L. Lions. Optimal Control of Systems Governed by Partial Differential Equations. Springer-Verlag, 1971.

[16] N. Papadakis, T. Corpetti, and E. Mémin. Dynamically consistent optical flow estimation. In Proceedings of In-

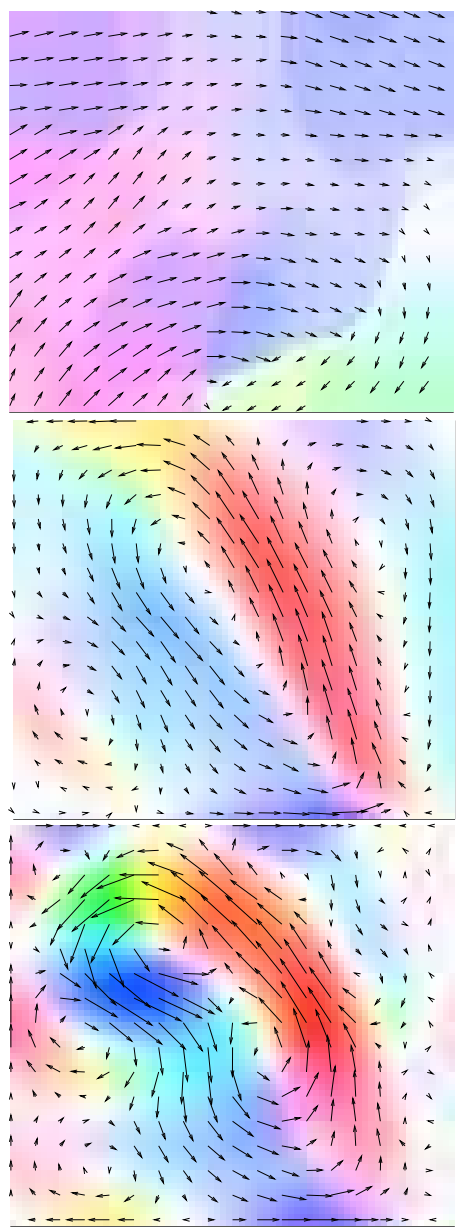

Figure 13. Results of motion estimation. Top: Sun et al. [18]. Middle: full model. Bottom: our method.

ternational Conference on Computer Vision (ICCV), Rio de Janeiro, Brazil, Oct. 2007.

[17] M. Restelli, L. Bonaventura, and R. Sacco. A semiLagrangian discontinuous Galerkin method for scalar advection by incompressible flows. Journal of Computational Physics, 216(1):195-215, 2006.

[18] D. Sun, S. Roth, and M. Black. Secrets of optical flow estimation and their principles. In Proceedings of European Conference on Computer Vision (ECCV), pages 2432-2439, 2010.

[19] D. Suter. Motion estimation and vector splines. In Proceedings of International Conference on Computer Vision and Pattern Recognition (CVPR), pages 939-942, 1994.

[20] O. Titaud, A. Vidard, I. Souopgui, and F.-X. Le Dimet. Assimilation of image sequences in numerical models. Tellus A, 62:30-47, 2010.

[21] E. Valur Hólm. Lectures notes on assimilation algorithms. Technical report, European Centre for MediumRange Weather Forecasts Reading, U.K, Apr. 2008. 